\title{
CONTINUUM-WISE EXPANSIVE DIFFEOMORPHISMS
}

\author{
KAZUHiRo SAKaI
}

\begin{abstract}
In this paper, we show that the $C^{1}$ interior of the set of all continuum-wise expansive diffeomorphisms of a closed manifold coincides with the $C^{1}$ interior of the set of all expansive diffeomorphisms. And the $C^{1}$ interior of the set of all continuum-wise fully expansive diffeomorphisms on a surface is investigated. Furthermore, we have necessary and sufficient conditions for a diffeomorphism belonging to these open sets to be Anosov.
\end{abstract}

In the investigation of the structural stability of differentiable dynamical systems and its relationship to the hyperbolic structure, a number of fundamental topological properties were discovered. One of the most important notion of the stability theory of dynamical system is an expansiveness and which is still studied by several resarchers in the context of general topology.

Let $X$ be a compact metric space with metric $d$ and let $f: X \rightarrow X$ be a homeomorphism. We say that $f$ is expansive if there is a number $e \geq 0$ such that for every $x, y \in X(x \neq y)$, there is $n=n(x, y) \in \mathbf{Z}$ satisfying $d\left(f^{n}(x), f^{n}(y)\right) \geq e$, and this notion was generalized by Kato [5]. He introduced a notion of continuum-wise expansive homeomorphisms there and many theorems concerning expansive homeomorphisms were generalized to the case of continuum-wise expansive homeomorphisms. We say that $f$ is continuum-wise expansive if there is a number $e \geq 0$ such that for every nondegenerate subcontinuum of $A \subset X$, there is $n=n(A) \in \mathbf{Z}$ satisfying $\operatorname{diam} f^{n}(A) \geq e$. Here $\operatorname{diam} S=\sup \{d(x, y): x, y \in S\}$. Clearly every expansive homeomorphism is continuum-wise expansive but its converse is not true (see [5, Example 3.5]). In this paper, we

Keywords. Expansive, continuum-wise expansive, Anosov, quasi-Anosov, shadowing property, structural stability.

1991 Mathematics subject classifications: Primary: 54H20, 58F10, 58F15. 
investigate the dynamics of continuum-wise expansive diffeomorphisms from the differentiable viewpoint.

Let $M$ be a $C^{\infty}$ closed manifold and let $\operatorname{Diff}(M)$ be the space of $C^{1}$ diffeomorphisms of $M$ endowed with $C^{1}$ topology. Let $\mathcal{E}(M)$ and $\mathcal{C} \mathcal{E}(M)$ be the set of all expansive diffeomorphisms and the set of all continuumwise expansive diffeomorphisms in $\operatorname{Diff}(M)$ respectively.

Theorem 1. The $C^{1}$ interior of $\mathcal{C} \mathcal{E}(M), \operatorname{int} \mathcal{C E}(M)$, coincides with the $C^{1}$ interior of $\mathcal{E}(M)$, int $\mathcal{E}(M)$.

The notion of quasi-Anosov diffeomorphisms on $M$ was introduced by Mañé [9] and it was proved in [8] that $f \in \operatorname{int} \mathcal{E}(M)$ if and only if $f$ is a quasi-Anosov diffeomorphism. Clearly if $f$ is quasi-Anosov, then $f \in \operatorname{int} \mathcal{C E}(M)$. Our theorem will be obtained by showing that if $f \in \operatorname{int} \mathcal{C E}(M)$, then $f$ is quasi-Anosov.

Let $(X, d)$ be as before and assume that $X$ is a continuum. A homeomorphism $f: X \rightarrow X$ is called continuum-wise fully expansive ([6]) provided that for every $\varepsilon>0$ and $\delta>0$, there is a natural number $N=N(\varepsilon, \delta)>0$ such that if $A$ is a subcontinuum of $X$ with $\operatorname{diam} A \geq \delta$, then either $d_{H}\left(f^{n}(A), X\right)<\varepsilon$ for all $n \geq N$, or $d_{H}\left(f^{-n}(A), X\right)<\varepsilon$ for all $n \geq N$. Here $d_{H}$ denotes the Hausdorff metric. It is clear that every continuume-wise fully expansive homeomorphism is continuume-wise expansive but its converse is not true (see [6, Example 4.9]). Remark that there is an example [6, Example 2.2] of continuum-wise fully expansive homeomorphisms that is not expansive. The notion of continuum-wise fully expansive is closely related to that of topologically mixing. Indeed, it is proved in [6] that if $f: X \rightarrow X$ is continuum-wise fully expansive, then $f$ is topologically mixing.

Let we denote the set of all continuum-wise fully expansive diffeomorphisms of $M$ by $\mathcal{C F E}(M)$ and denote by $\operatorname{int} \mathcal{C F E}(M)$ its $C^{1}$ interior. Then we have the following

Corollary. When $\operatorname{dim} M=2$, $\operatorname{int} \mathcal{C E}(M)$ is equal to $\operatorname{int} \mathcal{C} \mathcal{F} \mathcal{E}(M)$.

This corollary is an easy consequence of Theorem 1. Indeed, clearly $\operatorname{int} \mathcal{C F E}(M) \subset \operatorname{int} \mathcal{C} \mathcal{E}(M)$. By Theorem $1 \operatorname{int} \mathcal{C E}(M)$ is equal to the set of all quasi-Anosov diffeomorphisms. Since every quasi-Anosov diffeomorphism $f$ is Anosov when $\operatorname{dim} M=2$ (see [9]) and since every Anosov diffeomorphism is topologically conjugate to a hyperbolic toral automorphism when $\operatorname{dim} M=2, f$ is continuum-wise fully expansive (see [6, Proposition 2.4]).

Let $M$ be as before and let $d$ be a metric on $M$ induced from a Riemannian metric $\|\cdot\|$ on $T M$. A sequence $\left\{x_{k}\right\}_{k=a}^{b}(-\infty \leq a<b \leq \infty)$ of 
points is called a $\delta$-pseudo-orbit of $f \in \operatorname{Diff}(M)$ if $d\left(f\left(x_{k}\right), x_{k+1}\right)<\delta$ for $a \leq k \leq b-1$. Given $\varepsilon>0,\left\{x_{k}\right\}_{k=a}^{b}$ is said to be $\varepsilon$-shadowed by $x \in M$ if $d\left(f^{k}(x), x_{k}\right)<\varepsilon$ for $a \leq k \leq b$. We say that $f$ has the shadowing property if for $\varepsilon>0$ there is $\delta>0$ such that every $\delta$-pseudo-orbit of $f$ can be $\varepsilon$-shadowed by some point.

Let us denote by $\mathcal{H}(M)$ the set of all homeomorphisms of $M$ endowed with $C^{0}$ topology. We say that $f$ is structurally stable if there is a $C^{1}$ neighborhood $\mathcal{U}(f) \subset \operatorname{Diff}(M)$ such that for every $g \in \mathcal{U}(f)$, there is $h \in \mathcal{H}(M)$ satisfying $g \circ h=h \circ f$. We say that $f$ is topologically stable if for every $\varepsilon>0$, there is a $C^{0}$ neighborhood $\mathcal{U}_{\varepsilon}(f) \subset \mathcal{H}(M)$ such that for every $g \in \mathcal{U}_{\varepsilon}(f)$, there is a continuous map $h: M \rightarrow M$ satisfying $f \circ h=h \circ g$ and $d(h, i d)<\varepsilon$. We remark that since $M$ is a manifold, $d(h, i d)<\varepsilon$ implies $h(M)=M$ for a sufficently small $\varepsilon$. It is well known that every structurally stable diffeomorphism is topologically stable $([\mathbf{1 2}])$ but its converse is not true, and that every topologically stable diffeomorphism has the shadowing property $([\mathbf{1 1}])$. The author do not know that whether every diffeomorphism having the shadowing property is topologically stable (cf. [14]).

We say that $f \in \operatorname{Diff}(M)$ is persistent $([\mathbf{7}])$ if for each $\varepsilon>0$, there is $\delta>0$ such that for every $x \in M$ and $g \in \mathcal{H}(M)$ with $d(f, g)<\delta$, there is $y \in M$ satisfying $d\left(f^{n}(x), g^{n}(y)\right)<\varepsilon(\forall n \in \mathbf{Z})$. It is easy to see that every topologically stable diffeomorphism $f$ on $M$ is persistent. Notice that every pseudo-Anosov diffeomorphism $f$ on a surface is persistent (see [7, Corollary 3.1]) but $f$ does not have the shadowing property so that $f$ is not topologically stable. These notions are independent of metrics for $M$ and are conjugacy invariant.

Clearly every Anosov diffeomorphism is contained in $\operatorname{int} \mathcal{C} \mathcal{E}(M)$, however, there exists $f \in \operatorname{int} \mathcal{C} \mathcal{E}(M)$ that is not Anosov when $\operatorname{dim} M=3$ (see $[\mathbf{3}]$ ). In this paper, under the above notations, we have the following

Theorem 2. Let $f \in \operatorname{int} \mathcal{C E}(M)$. Then the following conditions are mutually equivalent.

(1) $f$ is Anosov

(2) $f$ is structurally stable

(3) $f$ has the shadowing property

(4) $f$ is topologically stable

(5) $f$ is persistent.

\section{Proofs of Theorems}

It was also proved in $[\mathbf{9}$, Theorem $\mathrm{A}]$ that $f \in \operatorname{Diff}(M)$ is quasi-Anosov if and only if $f$ satisfies Axiom A and for every $x \in M$ we have 


$$
T_{x} W^{s}(x) \cap T_{x} W^{u}(x)=\left\{0_{x}\right\},
$$

where $W^{s}(x)$ and $W^{u}(x)$ are the stable manifold and the unstable manifold of $x \in M$ respectively. Hereafter, let $\mathcal{Q A}(M) \subset \operatorname{Diff}(M)$ be the set of all quasi-Anosov diffeomorphisms. As we stated before Mañé proved that $\mathcal{Q A}(M)=\operatorname{int} \mathcal{E}(M)$, and thus, to prove Theorem 1 it is enough to show that $\operatorname{int} \mathcal{C} \mathcal{E}(M) \subset \mathcal{Q A}(M)$.

Our proof of this theorem rely largely on the result which was proved by Aoki $[\mathbf{1}]$ and Hayashi $[4]$ independently. Let $P(f)$ denote the set of all periodic points of $f \in \operatorname{Diff}(M)$, and let $\mathcal{F}(M)$ be the set of all $f \in \operatorname{Diff}(M)$ having a $C^{1}$-neighborhood $\mathcal{U}(f) \subset \operatorname{Diff}(M)$ such that every $p \in P(g)(\forall g \in \mathcal{U}(f))$ is hyperbolic. Then such a set was characterized as the set of all diffeomorphisms satisfying Axiom A with no-cycles.

Theorem 1 will be proved by using the following proposition. This result is already stated in $[\mathbf{8}$, Lemma 3] for $\operatorname{int} \mathcal{E}(M)$, and its proof is almost the same as that of [2, Proof of Theorem 1]. Here we shall give a rather simple proof for the completeness.

Proposition. The $C^{1}$ interior of $\mathcal{C E}(M), \operatorname{int} \mathcal{C}(M)$, is a subset of $\mathcal{F}(M)$.

Proof: Let $f \in \operatorname{int} \mathcal{C E}(M)$. To get the conclusion, it is enough to show that every $p \in P(f)$ is hyperbolic.

Fix a neighborhood $\mathcal{U}(f) \subset \operatorname{int} \mathcal{C E}(M)$ of $f$, and by assuming that there is a non-hyperbolic periodic point $p=f^{n}(p)$, we shall derive a contradiction. Here $n>0$ is the prime period of $p$. The tangent space $T_{p} M$ splits into the direct sum $T_{p} M=E_{p}^{u} \oplus E_{p}^{s} \oplus E_{p}^{c}$ where $E_{p}^{u}, E_{p}^{s}$ and $E_{p}^{c}$ are $D_{p} f^{n}$-invariant subspaces corresponding to the absolute values of the eigenvalues of $D_{p} f^{n}$ with greater than one, less than one and equal to one, and suppose $E_{p}^{c} \neq 0$. Then, for every $\varepsilon>0$ there exists a linear automorphism $\mathcal{O}: T_{p} M \rightarrow T_{p} M$ such that

$$
\begin{cases}\|\mathcal{O}-I\| \leq \varepsilon, & \\ \mathcal{O}\left(E_{p}^{\sigma}\right)=E_{p}^{\sigma} & \text { for } \sigma=s, u \text { and } c \\ & \text { all eigenvalues of } \mathcal{O} \circ D_{p} f_{\mid E_{p}^{c}}^{n} \text { are of a root of unity }\end{cases}
$$

where $I: T_{p} M \rightarrow T_{p} M$ is an identity map. By making use of Franks's Lemma (see [2, Lemma 1.1]), we can find $\delta_{0}>0$ and $g \in \mathcal{U}(f)$ such that

(i) $B_{4 \delta_{0}}\left(f^{i}(p)\right) \cap B_{4 \delta_{0}}\left(f^{j}(p)\right)=\phi$ for $0 \leq i \neq j \leq n-1$,

(ii) $g(x)=f(x)$ for $x \in\left\{p, f(p), \ldots, f^{n-1}(p)\right\} \cup\left\{M \backslash \cup_{i=0}^{n-1} B_{4 \delta_{0}}\left(f^{i}(p)\right)\right\}$, 
(iii) $g(x)=\exp _{f^{i+1}(p)} \circ D_{f^{i}(p)} f \circ \exp _{f^{i}(p)}^{-1}(x)$ for $x \in B_{\delta_{0}}\left(f^{i}(p)\right)$ $(0 \leq i \leq n-2)$,

(iv) $g(x)=\exp _{p} \circ \mathcal{O} \circ D_{f^{n-1}(p)} f \circ \exp _{f^{n-1}(p)}^{-1}(x)$ for $x \in B_{\delta_{0}}\left(f^{n-1}(p)\right)$, where $B_{\varepsilon}(x)=\{y \in M \mid d(x, y) \leq \varepsilon\}$ for $\varepsilon>0$. Notice that $g$ is continuum-wise expansive.

Define $G=\mathcal{O} \circ D_{p} f^{n}$. Then there exists $m>0$ and $v \in E_{p}^{c} \backslash\left\{0_{p}\right\}$ such that $\|v\|=1$ and $G^{m}(t v)=t v(t \geq 0)$. For a sufficently small $0<\delta_{1}<\delta_{0}$, we have

$$
g_{\mid \exp _{p} T_{p} M\left(\delta_{1}\right)}^{m n}=\exp _{p} \circ G^{m} \circ \exp _{p}^{-1}
$$

where $T_{p} M\left(\delta_{1}\right)=\left\{v \in T_{p} M \mid\|v\| \leq \delta_{1}\right\}$. Put $v_{\delta_{1}}=\delta_{1} \cdot v \in E_{p}^{c} \cap T_{p} M\left(\delta_{1}\right)$. Then it is clear that

$$
g_{\mid \exp _{p} v_{\delta_{1}}}^{m n}=i d_{\mid \exp _{p} v_{\delta_{1}}} .
$$

This is a contradiction since $\exp _{p} v_{\delta_{1}}$ is a continuum.

To prove Theorem 1 we shall prepair some notations. Let $f \in \operatorname{Diff}(M)$ satisfy Axiom A. Thus the non-wandering set of $f, \Omega(f)$, is hyperbolic. For any $\varepsilon>0$ and for $x \in \Omega(f)$, the local stable manifold and the local unstable manifold are denoted by $W_{\varepsilon}^{s}(x)$ and $W_{\varepsilon}^{u}(x)$ respectively. We may assume that there are $\varepsilon_{0}>0$ and $0<\lambda<1$ such that for $x \in \Omega(f)$

$$
\begin{aligned}
d\left(f^{n}(y), f^{n}(z)\right) & \leq \lambda^{n} d(y, z) & & \text { for } y, z \in W_{\varepsilon_{0}}^{s}(x), \\
d\left(f^{-n}(y), f^{-n}(z)\right) & \leq \lambda^{n} d(y, z) & & \text { for } y, z \in W_{\varepsilon_{0}}^{u}(x) .
\end{aligned}
$$

It is well known that $M=\cup_{x \in \Omega(f)} W^{\sigma}(x)(\sigma=s, u)$, where

$$
\begin{aligned}
W^{s}(x) & =\cup_{n \geq 0} f^{-n} W_{\varepsilon_{0}}^{s}\left(f^{n}(x)\right), \\
W^{u}(x) & =\cup_{n \geq 0} f^{n} W_{\varepsilon_{0}}^{u}\left(f^{-n}(x)\right) .
\end{aligned}
$$

Proof of Theorem 1: Let $f \in \operatorname{int} \mathcal{C E}(M)$. Then $f$ satisfy Axiom A by the proposition. We shall show that for every $x \in M$

$$
T_{x} W^{s}(x) \cap T_{x} W^{u}(x)=\left\{0_{x}\right\} .
$$

This equality will be proved by using a standard perturbation procedure.

For $f \in \operatorname{int} \mathcal{C E}(M)$, by assuming that there is $x \in M \backslash \Omega(f)$ such that $E(x)=T_{x} W^{s}(x) \cap T_{x} W^{u}(x) \neq\left\{0_{x}\right\}$, we shall derive a contradiction. For $\delta>0$, we denote the connected component of $0_{x}$ in $T_{x} M(\delta) \cap E(x)$ by $E_{\delta}(x)$. Fix any neighborhood $\mathcal{U}(f) \subset \operatorname{int} \mathcal{C} \mathcal{E}(M)$ of $f$. Then, since $T_{x} \exp _{x} E_{\delta}(x)=E(x)$, we can construct two diffeomorhisms $\varphi_{1}, \varphi_{2}$ : $M \rightarrow M$ such that 


$$
\left\{\begin{array}{l}
\varphi_{i}(x)=x \quad(i=1,2) \\
\varphi_{i \mid M \backslash B_{\delta_{0}}(x)}=i d \quad(i=1,2) \\
\varphi_{1}\left(\exp _{x} E_{\delta_{0} / 4}(x)\right) \subset W^{s}(x), \varphi_{2}\left(\exp _{x} E_{\delta_{0} / 4}(x)\right) \subset W^{u}(x) \\
f \circ \varphi_{1}^{-1}, \varphi_{2} \circ f \in \mathcal{U}(f),
\end{array}\right.
$$

where $0<\delta_{0}<\varepsilon_{0}$ is sufficiently small. Put

$$
g(y)= \begin{cases}f(y) & \text { if } y \notin B_{\delta_{0}}(x) \cup B_{\delta_{0}}\left(f^{-1}(x)\right) \\ f \circ \varphi_{1}^{-1}(y) & \text { if } y \in B_{\delta_{0}}(x) \\ \varphi_{2} \circ f(y) & \text { if } y \in B_{\delta_{0}}\left(f^{-1}(x)\right) .\end{cases}
$$

Then $g \in \mathcal{U}(f)$ so that $g$ is continuum-wise expansive with constant $e>0$. However, by the hyperbolicity, it is easy to see that there is $0<\delta<\delta_{0} / 4$ such that $\operatorname{diam} g^{n}\left(\exp _{x} E_{\delta}(x)\right)<e$ for $n \in \mathbf{Z}$. This is a contradiction and so we have $f \in \mathcal{Q} \mathcal{A}(M)$.

To prove Theorem 2 we shall use the following two lemmas.

Lemma 1. Let $f \in \mathcal{Q A}(M)$. Then there is a constant $c>0$ and a $C^{1}$ neighborhood $\mathcal{U}(f) \subset \operatorname{Diff}(M)$ such that for every $g \in \mathcal{U}(f)$, if $d\left(g^{n}(x), g^{n}(y)\right) \leq c(n \in \mathbf{Z})$, then $x=y$.

Proof: See [13, proof of theorem].

Lemma 2. Let $g: M \rightarrow M$ be an expansive homeomorphism with constant $c>0$. Then for every $\alpha>0$, there is an integer $N=N(g, \alpha)>0$ such that $d\left(g^{n}(x), g^{n}(y)\right) \leq c$ for all $-N \leq n \leq N$ implies $d(x, y)<\alpha$.

Proof: See $[\mathbf{1 0}$, p. 318, Lemma II].

Proof of Theorem 2: The equivalence of (1), (2) and (3) follows from $[\mathbf{9}$, Corollary 1] and [13, Theorem]. To get the conclusion it is enough to show an implication $(5) \rightarrow(2)$ because $(2) \rightarrow(4) \rightarrow(5)$ is well known. Hereafter let $f \in \mathcal{Q A}(M)$ has a persistency, and let $c>0$ and $\mathcal{U}(f)$ be as in Lemma 1. Fix $0<\varepsilon<c / 2$ and let $\mathcal{U}_{\varepsilon}(f)$ be a $C^{0}$ neighborhood of $f$ as in the definition of a persistency.

For any $g \in \mathcal{U}_{\varepsilon}(f) \cap \mathcal{U}(f)$ and $x \in M$, there is a unique point $y \in M$ such that $d\left(f^{n}(x), g^{n}(y)\right)<\varepsilon(n \in \mathbf{Z})$. For, if there exists $y^{\prime}(\neq y) \in M$ such that $d\left(f^{n}(x), g^{n}\left(y^{\prime}\right)\right)<\varepsilon(n \in \mathbf{Z})$, then

$d\left(g^{n}(x), g^{n}\left(y^{\prime}\right)\right) \leq d\left(g^{n}(y), f^{n}(x)\right)+d\left(f^{n}(x), g^{n}\left(y^{\prime}\right)\right)<2 \varepsilon<c(n \in \mathbf{Z})$. 
Thus $y=y^{\prime}$ by Lemma 1 and which is a contradiction.

We denote such $y$ by $h_{g}(x)$ for $x \in M$. Then $h_{g}: M \rightarrow M$ is a map such that $h \circ f=g \circ h$. Indeed, since $d\left(f^{n}(x), g^{n}\left(h_{g}(x)\right)\right)<\varepsilon(n \in \mathbf{Z})$, we have

$$
d\left(f^{n}(f(x)), g^{n}\left(h_{g}(f(x))\right)\right)<\varepsilon \text { for } n \in \mathbf{Z}
$$

and

$$
d\left(f^{n}(f(x)), g^{n}\left(g\left(h_{g}(x)\right)\right)\right)<\varepsilon \text { for } n \in \mathbf{Z} .
$$

Thus, for $n \in \mathbf{Z}$

$$
\begin{aligned}
d\left(g^{n}\left(h_{g} \circ f(x)\right), g^{n}\left(g \circ h_{g}(x)\right)\right) & \leq d\left(g^{n}\left(h_{g} \circ f(x)\right), f^{n+1}(x)\right) \\
& +d\left(f^{n+1}(x), g^{n}\left(g \circ h_{g}(x)\right)\right) \leq 2 \varepsilon<c .
\end{aligned}
$$

From this $h \circ f=g \circ h$ is concluded. Clearly we have $d\left(h_{g}(x), x\right)<\varepsilon$ for $x \in M$.

To prove the continuity of $h_{g}$, for every $\alpha>0$, let $N=N(g, \alpha)>0$ be as in Lemma 2. Since $f$ is uniformly continuous, there is $\beta>0$ such that $d(x, y)<\beta(x, y \in M)$ implies $d\left(f^{n}(x), f^{n}(y)\right) \leq c-2 \varepsilon$ for $-N \leq n \leq N$. Thus

$$
\begin{aligned}
d\left(g^{n} \circ h_{g}(x), g^{n} \circ\right. & \left.h_{g}(y)\right) \leq d\left(g^{n} \circ h_{g}(x), f^{n}(x)\right)+d\left(f^{n}(x), f^{n}(y)\right) \\
& +d\left(f^{n}(y), g^{n} \circ h_{g}(y)\right)<2 \varepsilon+d\left(f^{n}(x), f^{n}(y)\right) \leq c
\end{aligned}
$$

for $-N \leq n \leq N$. Hence we have $d\left(h_{g}(x), h_{g}(y)\right)<\alpha$.

Since

$$
\begin{array}{r}
d\left(f^{n}(x), f^{n}(y)\right) \leq d\left(f^{n}(x), g^{n} \circ h_{g}(x)\right)+d\left(g^{n} \circ h_{g}(x), g^{n} \circ h_{g}(y)\right) \\
+d\left(g^{n} \circ h_{g}(y), f^{n}(y)\right)<2 \varepsilon+d\left(g^{n} \circ h_{g}(x), g^{n} \circ h_{g}(y)\right)
\end{array}
$$

if we assume that $h_{g}(x)=h_{g}(y)$, then $d\left(f^{n}(x), f^{n}(y)\right)<c$ for all $n \in \mathbf{Z}$. Thus $x=y$ and so $f$ is structurally stable. The proof of Theorem 2 is completed.

\section{References}

1. N. Aoki, The set of Axiom A diffeomorphisms with no cycle, Bol. Soc. Brasil. Mat. (N.S.) 23 (1992), 21-65.

2. J. FRANKS, Necessary conditions for stability of diffeomorphisms, Trans. Amer. Math. Soc. 158 (1971), 301-308.

3. J. Franks And C. Robinson, A quasi-Anosov diffeomorphisms that is not Anosov, Trans. Amer. Math. Soc. 223 (1976), 267-278. 
4. S. HaYAshi, Diffeomorphisms in $\mathcal{F}^{1}(M)$ satisfy Axiom A, Ergodic Theory Dynam. Systems 12 (1992), 233-253.

5. H. Kato, Continuum-wise expansive homeomorphisms, Canad. J. Math. 45 (1993), 576-598.

6. H. Kato, Concerning continuum-wise fully expansive homeomorphisms of continua, Topology Appl. 53 (1993), 239-258.

7. J. Lewowicz, Persistence in expansive systems, Ergodic Theory Dynam. Systems 3 (1983), 567-578.

8. R. MAÑé, Expansive Diffeomorphisms, in "Dynamical SystemsWarwick 1974," (A. Manning, ed.), Lecture Notes in Math. 468, Springer, 1975, pp. 162-174.

9. R. MaÑé, Quasi-Anosov Diffeomorphisms and Hyperbolic Manifolds, Trans. Amer. Math. Soc. 229 (1977), 351-370.

10. R. MAÑÉ, Expansive homeomorphisms and topological dimension, Trans. Amer. Math. Soc. 252 (1979), 313-319.

11. P. Walters, On the pseudo orbit tracing property and its relationship to stability, in "The Structure of Attractors in Dynamical Systems," Lecture Notes in Math. 668, Springer, 1974, pp. 231-244.

12. C. Robinson, Stability theorems and hyperbolicity in dynamical systems, Rocky Mountain J. Math. 7 (1977), 425-437.

13. K. SAKAI, Quasi-Anosov Diffeomorphisms and Pseudo orbit tracing property, Nagoya Math. J. 111 (1988), 111-114.

14. K. YAno, Topologically stable homeomorphisms of the circle, Nagoya Math. J. 79 (1980), 145-149.

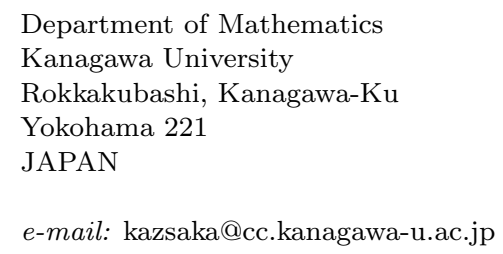

Primera versió rebuda el 20 de Desembre de 1995, darrera versió rebuda el 21 de Juliol de 1997 